\title{
Bioanalysis
}

\section{Chemical derivatization in bioanalysis}

\author{
"This themed issue covers advances in existing derivatization techniques used \\ in bioanalytical research, as well as innovative new methods and approaches..."
}

Keywords: chemical derivatization $\bullet$ chiral analysis $\bullet$ chromatographic separation $\bullet$ ionization efficiency $\bullet$ polar compounds $\bullet$ selectivity

\section{Applications}

Chemical derivatization has long proved itself as an analytical technique in bioanalysis to overcome problems associated with low ionization efficiency, compound instability, poor selectivity or unacceptable chromatographic performance (poor retention, bad peak shape and carryover issues) and even poor volatility for GC separation $[1,2]$. This technique is a powerful tool in many areas of chemistry including medical, forensic, food science, doping control and environmental disciplines. The goal of chemical derivatization is to modify the structure of the analyte (either a nucleophile or electrophile) using a chemical reagent (either an electrophile or nucleophile depending on the nature of the analyte) and, as a result, a new compound (the reaction's derivate) with improved chemical and physical properties for analysis is formed. The reaction conditions (amount of the reagent, reaction time and temperature, etc) are optimized in favor of the formation of the desired derivative with the highest possible reaction yield. Additional sample clean up procedures can be developed to eliminate unwanted byproducts and excess reagents, thereby minimizing analyte inferences upon analysis.

Using chemical derivatization, analysis of the impossible becomes possible. Many examples of this have been presented in the literature; impacting GC, LC-MS/MS [1-3] and NMR [4-6] detection. Most notable has been the chromatographic separation of enantiomers through chiral derivatization
\end{abstract}

using specific resolving reagents without the use of specialized chiral columns and separation conditions [7-9].

\section{Considerations}

The selection of the appropriate chemical reagent is essential for a successful derivatization and is dependent on the specific application. In general, if the target analyte is a nucleophile (compound with an excess of electrons), an electrophile (compounds with overall electron deficiency) is selected as reagent, and vice versa. Reagents need to be selective (target one specific site of molecule), thereby avoiding derivatization at multiple sites in the target molecule, metabolites or endogenous components. As an example, for a molecule containing both hydroxyl and amino functional groups the use of acid chlorides or anhydrides as derivitization reagents should be avoided as these will derivatize both functional groups. On the contrary, the use of dansyl chloride as a derivitization reagent is appropriate for amino and phenol functional groups as it does not react with aliphatic alcohols. Other requisites to be considered in the selection of the reagent include availability (commercially), purity and cost. Typically, the cost of reagents is minimal, and therefore does not represent a barrier for use.

Using optimized conditions, chemical derivatization procedures are typically robust enough to be applied for pharmaceutical bioanalysis, and are capable of meeting regulatory expectations. This is usually demonstrated during a rigorous validation

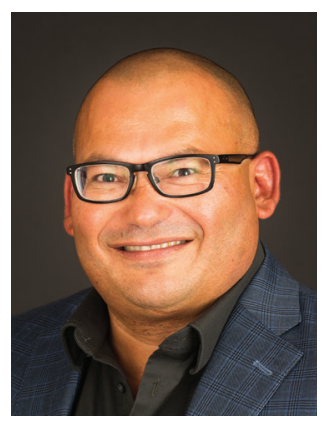

Hermes Licea Perez Author for correspondence: Bioanalytical Sciences \& Toxicokinetics, PTS-DMPK, GlaxoSmithKline Pharmaceuticals, 709 Swedeland Road King of Prussia, PA 19406, USA hermes.2.licea-perez@gsk.com

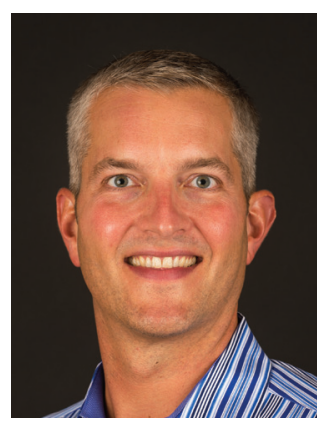

Christopher A Evans Bioanalytical Sciences \& Toxicokinetics, PTS-DMPK, GlaxoSmithKline Pharmaceuticals, 709 Swedeland Road, King of Prussia, PA 19406, USA 
process where several parameters, including but not limited to, accuracy, precision, selectivity, matrix effect, etc. The selection of internal standard is essential to correct for any possible analyte loss during the various steps of sample handling and bioanalysis; thus ensuring robustness of the assay. When possible, a deuterium or ${ }^{13} \mathrm{C}$ stable internal standard should be used, otherwise an analog with similar reactivity, recovery, and chromatographic properties could be substituted. Also, it is imperative to consider and evaluate, where possible, metabolic pathways of the analyte of interest; conversion of metabolites back to parent molecule need to be avoided during the derivatization procedure, as these processes often involve harsh conditions ( $\mathrm{pH}$, heat, long incubation times, etc.). Unfortunately, this can be complicated by the lack of reference standards for the metabolites, and the lack of metabolic information early in the drug development lifecycle due to differential or accelerated development strategies.

\section{Chemical derivatization as an art form}

The use of chemical derivatization has declined in recent years as new separation technologies have evolved and become more commonplace. The evolution of supercritical fluid chromatography (SFC), for example, has opened a new avenue for chiral stereoisomeric analysis; thereby reducing the need for chiral derivatization in certain instances [10]. More sensitive generations of triple quadrupole mass spectrometric instrumentation with novel or improved ionization technologies are pushing the detection limits to low picogram levels, and as result the demand for chemical derivatization to improve assay sensitivity (through improved ionization or selectivity) have decreased. Other technologies including UHPLC; micro-/nano-LC (for better ionization efficiency); TOF instruments with ion mobility capability (electronic separation, rather than chemical/physical) have also contributed to the decline of chemical derivitization in the bioanalytical laboratory. With that said however, the technique is still applied for very complex separations where aforementioned technologies cannot make an adequate impact. Sometimes coupling chemical derivatization with one of these technologies has an enhanced/additive impact. In particular, combination of SFC with chiral derivatization has shown to be superior for chiral separation as compared with that SFC analysis (data not shown).

Due to this decline of the technique and its complexity as compared with other analytical techniques, chemical derivatization has evolved into a specialty 'art form' in the laboratory, requiring specialized skills combined with a strong chemistry proficiency.
As a consequence, fewer scientists in DMPK environments succeed to master the technique and become proficient in its application. The question then becomes how to preserve these skills and pass them on to the future generations of analytical scientists. Special issues such as this, review articles, book chapters, guidance containing experimental protocols will hopefully facilitate and promote the use of chemical derivatization as great analytical tool.

\section{Outline}

This themed issue covers advances in existing derivatization techniques used in bioanalytical research, as well as innovative new methods and approaches (e.g., combination of derivatization with microflow LC-MS and the idea of new chemical tagging techniques by Niwa et al. [11]).

The issue aims to cover aspects related to:

- Derivatization methods in LC-MS bioanalysis (including HPLC);

- Peptide derivatization for analysis of protein therapeutics;

- Chiral derivatization reagents applied to biological samples (see Vashistha et al. [12]);

- Derivatization for analysis of endogenous compounds (see 'Beyond Classical Derivatization: Analyte 'derivatives' in the bioanalysis of endogenous and exogenous compounds' by Barnaby et al. [13], or 'Derivatization of steroids in biological samples for GC-MS and LC-MS analyses' by Marcos et al. [14]);

- Derivatization procedures in human doping control (see interesting review by Athanasiadou et al. [15]).

While it is true that chemical derivatization is just another tool in the bioanalytical toolbox, it is a 'must have' for a DMPK laboratory and one that will continue to have an impact addressing many bioanalytical challenges.

Therefore, if you don't like your analyte, change it (with chemical derivatization that is)!

\section{Financial \& competing interests disclosure}

The authors have no relevant affiliations or financial involvement with any organization or entity with a financial interest in or financial conflict with the subject matter or materials discussed in the manuscript. This includes employment, consultancies, honoraria, stock ownership or options, expert testimony, grants or patents received or pending, or royalties.

No writing assistance was utilized in the production of this manuscript 


\section{References}

Papers of special note have been highlighted as:

• of considerable interest

1 Knapp D. Handbook of Analytical Derivatization Reactions. John Wiley \& Sons, NY, USA (1979).

-• Highly recommended reference.

2 Handbook of Derivatives for Chromatography. Blau K, King GS (Eds). Heyden \& Sons, London, UK (1977).

3 Gas Chromatography (GC) Derivatization. Regis Chromatography Catalog. www.chromspec.com/pdf/e/rg01.pdf

4 Dale JA, Dull DL, Mosher HS. $\alpha$-Methoxy- $\alpha$ trifluoromethylphenylacetic acid, a versatile reagent for the determination of enantiomeric composition of alcohols and amines. J. Org. Chem. 34(9), 2543-2549 (1969).

5 Dale JA, Mosher HS. Nuclear magnetic resonance enantiomer regents. Configurational correlations via nuclear magnetic resonance chemical shifts of diastereomeric mandelate, $\mathrm{O}$-methylmandelate, and $\alpha$-methoxy- $\alpha$ trifluoromethylphenylacetate (MTPA) esters. J. Am. Chem. Soc. 95(2), 512-519 (1973).

6 Ward DE, Rhee CK. A simple method for the microscale preparation of Mosher's acid chloride. Tetrahedron Lett. 32(49), 7165-7166 (1991).

7 Chandrul KK, Srivastava B. Enantiomeric separation in pharmaceutical analysis: a chromatographic approach. J. Chem. Pharm. Res. 2(4) 923-934 (2010).
8 Porter WH. Resolution of chiral drugs. Pure Appl. Chem. 63(8), 1119-1122 (1991).

9 Görög S, Gazdag M. Enantiomeric derivatization for biomedical chromatography. J. Chromatogr. B. 659(1-2), 51-84 (1994)

10 Zhao Y, Woo G, Thomas S, Semin D, Sandra P. Rapid method development for chiral separation in drug discovery using sample pooling and supercritical fluid chromatographymass spectrometry. J. Chromatogr. A 1003(1-2), 157-166 (2003).

11 Niwa M, Miyuki Watanabe M, Watanabe N. Chemical derivatization in LC-MS bioanalysis: current and future challenges. Bioanalysis 7(19), 2443-2449 (2015).

12 Vashistha VK, Bhushan R. Bioanalysis and enantioseparation of DL-carnitine in human plasma by derivatization approach. Bioanalysis 7(19), 2477-2488 (2015).

13 Barnaby OS, Benitex Y, Cantone JL et al. Beyond classical derivatization: analyte 'derivatives' in the bioanalysis of endogenous and exogenous compounds. Bioanalysis 7(19), 2501-2513 (2015).

14 Marcos J, Pozo OJ. Derivatization of steroids in biological samples for GC-MS and LC-MS analyses. Bioanalysis 7(19), 2515-2536 (2015).

15 Athanasiadou I, Kiousi P, Kioukia-Fougia N, Lyris E, Angelis YS. Current status and recent advantages in derivatization procedures in human doping control. Bioanalysis 7(19), 2537-2556 (2015). 